ERRATUM

\title{
A quantitative Carleman estimate for second-order elliptic operators - ERRATUM
}

\author{
Ivica Nakić, Christian Rose and Martin Tautenhahn \\ https://doi.org/10.1017/prm.2018.55 Published online by Cambridge \\ University Press 27 December 2018 \\ Keywords: Carleman estimate; second order elliptic differential operator; explicit \\ weight function, ERRATUM
}

In the above article (Nakić, I., Rose, C., \& Tautenhahn, M. 2018) the affiliation for the author Martin Tautenhahn was listed incorrectly. The correct affiliation should be;

Technische Universität Chemnitz, Fakultät für Mathematik, Germany

This has been corrected in the online version of the article.

\section{Reference}

1 I. Nakić, C. Rose, and M. Tautenhahn (2018). A quantitative Carleman estimate for secondorder elliptic operators. Proc. R. Soc. Edinb. A., 1-24. doi:10.1017/prm.2018.55 